\title{
Sobre um novo tratamento da Leishmaniose
}

Assumpto extremamente arduo é o que vamos procurar realizar synthetizandio em um artigo a serie de experiencias que, debaixo da diresção scientifica do nosso prezado chefe Prof. A. Linderiberg, vamos realizando no serviço de Pelle e Syphilis da Sta. Casa, tentando, desse modo, encaminhar a solução desse magno problema, que é a cura ida Leishmaniose.

Impréssionado pelo numero sempre crescente de doentes que, atacados deste mal, invadem a nossa enfermaria, constituindo, já por isso, quasi que um serviço á parte, e onde teem uma permanencia por um tempo ainda mais impressionante, foi que, cheios de esperança, começamos a fazer o emprego do preparado Sb 211 de Bayer.

A afeção, entre nós conhecida por ulcera de Baurú, ferida brava, etc., a leishmaniose constitue verdadeiro entrave á penetração e ao progresso da civilização em certas zonas do nosso. Estado.

Multiplos teem sido os tratamentos aconselhados e experimentados para obtermos a cura dessa pathogenia, o que equivale dizer da pouca efficacia dos mesmos, não só sobre as lesões tegumentares, mas principalmente, sobre as das mucosas.

De todos os tratamentos usados até hoje, e que podemos, com Dutra e Silva, classificar em methodos de therapeutica expectante, de destruição das lesões e modernamente da chimiotherapia, nenhum tem entre nós preenchido o seu fim.

O emprego do primeiro methodo, embora tivesse partidarios como Schneider, Laveran e La Cava, deve ser combatido pois que a cura expontanea é excepcional e a generalização cutanea e mucosica facil.

O mesmo podemos dizer a respeito da destruição das partes lesadas, que se baseava na supposição de que o mal fosse superficial e exclusivamen- 


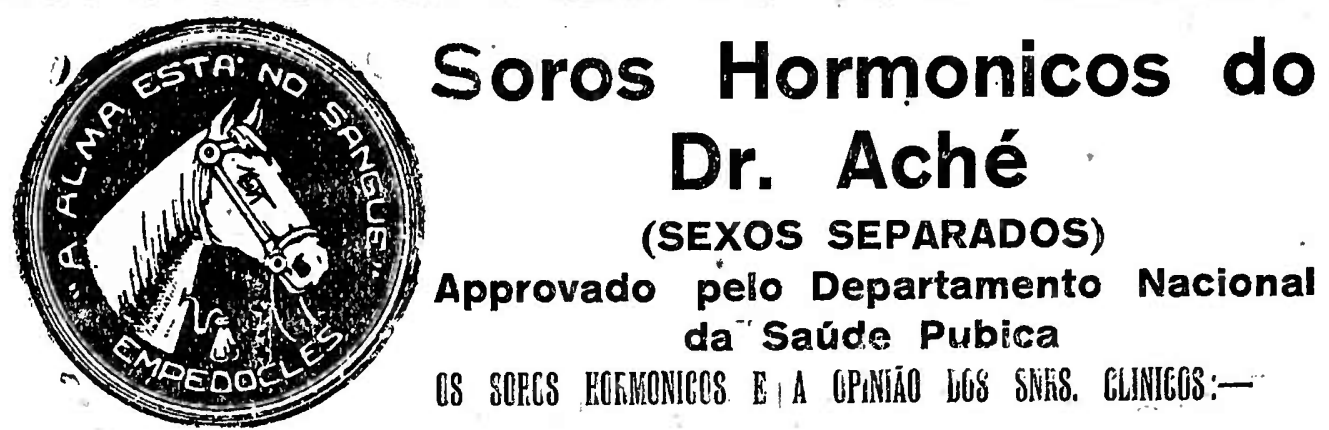

Dr. Adlauto Chastinet - Em 30-10-1925 - Rua 12 de Outubro n. 94 - São Paulo.

Añ observações obtidas com as applicações dos wôros prejarados pelo Laboratorio de Hormotherapia de Aché, Travasisos \& C., são as mais satisfactorias, especialmənte os sôroñ Hormomercurialino e Hormarsenicalino, de sexos separadors, que tenho tido a occasião de empregar, obtendo curas completas nos casos $\in \mathrm{m}$ que são indicados.

Dr. Carlos de Menezes - Em 11-1-1926 - Libero Baldaró n.. $\mathbf{3 7}$ - São Paulo.

Dou sempre preferencia em opotherapia aos preparados de Achié, Travassos \& Cia., dado o capricho da confecção e os resultados praticos que tenho colhido. Em um caso, recente, de hys-: terišmo com perturbação mental, fiquei realmente maravilhado com o resultado obtido com o Hormocerebrino.

Dr. Casisio Motta-Rua Barão de Itapetininga n. 52-São Paulo.

Tənho empregado frequentemente o sôro Hormonico, masculino e feminino, no tratamento das perturbações nervosas, com symptomas ás vezes de verdadeira neuraisthenia aguda, e conseguido rapido e duradoura melhora.

Dr. Floriano Bayma - Em 14-1-1926 - Rua Barão die Itapotininga n. 37-A - São Paulo.

... Tenho empregado com -successo as preparações do Laboratorio A.ché, Travassos \& Cia.

Dr. Mario Graccho - Em 18-1-1926 - Avénida Rangel Pestana São Paulo.

Os productos de Aché, Travassos \& Cia. rivalizam-se com os similares extrangeiror, pelo que devemos preferir aquelles, contribuindo assim para o bom nome do nosso Estado.

Dr. Oswaldo Pruissisegur - Em 8-81925 - Libero Badaró n. 53 - São Paulo.

Tenho obtido os melhores resultados com o emprego dos sôros Hormandrico e Hormogyno, em casos de laryngities chronicas.

Dr. Salvaldor lConti - Largo do Cambucy n. 55 - São Paulo.

Tenho o prazer de communicar-lhes que venho empregando, ha longa data, as suas ampolars de sôro Hormomercurialino, no tratamento da syphilis e suas manifestações. Pelas observiações colhidas e acompanhadars com todo o interesse, posso garantirlhes constituir esse seu preparado, um dos mais efficazes e de intieira confiança que conheço, para combater a lues e suas complicações ars mais graves.

\section{LABORATORIO DE HORMOTHERAPIA ACHE, TRAVESSOS \& CIA.-RIBEIRÃO PRETO} DEPOSITOS :--S. Paulo-Barão Itapetininga 65-Caixa 2843-Tel. Cid. 1938 Rio de Janeiro - Alfandega 95 - Caixa 1043 - Telephone Norte 6638 
te cutanec. Poderemos quando muito usal-o como methodo adjuvante naquelles casos em que haja exhuberancia de tecido, como nas formas verrucosas.

A acção chimiotropica positiva em relação aos protozoarios de certas substancias, veiu trazer esperanças a um grande numero de pesquizadores. Assim o 606 e o 914 foram entre nós usajdos por Dutra e Silva com acção pouco efficiente e bastante irregular, sendo completamente inactivos nas lesões das mucosas, muito embora no estrangeiro encontre defensores de altura de Erlich.

O remprego dos arsenicaes cresce hoje novamente de vulto, após a brilhante communicação feita pelo Prof. Aguiar Pupo, na Sociedade de Medicina e Cirurgia de S. Paulo, sobre o Eparseno.

Em Abril de 1912, Gaspar Vianna, por occasiãa do $2 .^{\circ}$ congresso de Medicina e Cirurgia em Bello Horizonte, fez uma communicação apresentando a cura definitiva do 1.0 doente de leishmaniose tegumentar pelo tartaro emetico. Estava, pois, feito o advento dos antimoniares na leishmaniose.

Entre os antimoniaes o tartaro emetico, o empregado por Vianna, é geralmente, senão o unico, usado pelos facultativos brasileiros.

Pelo facto de não curar todas as formas de lesões das mucosias, como pela sua intolerancia e perturbações que não raro produz, já não se fallando na sua morosidade de acção, ainda temos o problema insoluvel, o que tem levado, tanto no estrangeiro, como aqui, a serem usados novos preparados, mostrando a especificidade do antimonio nas lesões leishmanioticas.

Entre elles podemos citar o Protosan, preparado em Manguinhos por A. 'Machado e que o professor Lindenberg iniciou em experiencias em 1915, achando este antimonial mais efficaz que o emetico, porém sua inocuidade não está ainda perfeitamente estabelecida; o Disogo-luargol, que Oliveira Santiago acha muito efficaz nas lesões cutaneas e que os Profs. Lindenberg e Aguiar Pupo empregaram em doentes resistentes ao emetico, sem obterem resultados animadores; a Trioxidina, empregada pelo Prof. Lindenberg em imuitos doentes, tendo verificado ser esse medicamente especifico para a Leishmaniose ulcerosa, promovendo a rapida cicatrização das ulceras da pelle e das muccsas, mas que foi obrigado a abandonar pelos effeitos irritantes que produzem as injecções intramusculares, as quaes, além de muito dolorosas, produzem grandes infiltrados que suppuram; finalmente o mesmo Prof. Lindenberg, a 8 deste , levou 
á Sociedade de Biologia e Hygiene a communicação de experiencias fei- tas com novos preparados antimoniaes-Stibosam, Stibinyl e Antimosan, e na qual conclúe pela grande efficacia e absoluta inocuidade deste ultimo medicamente nas lesões cutaneas. .

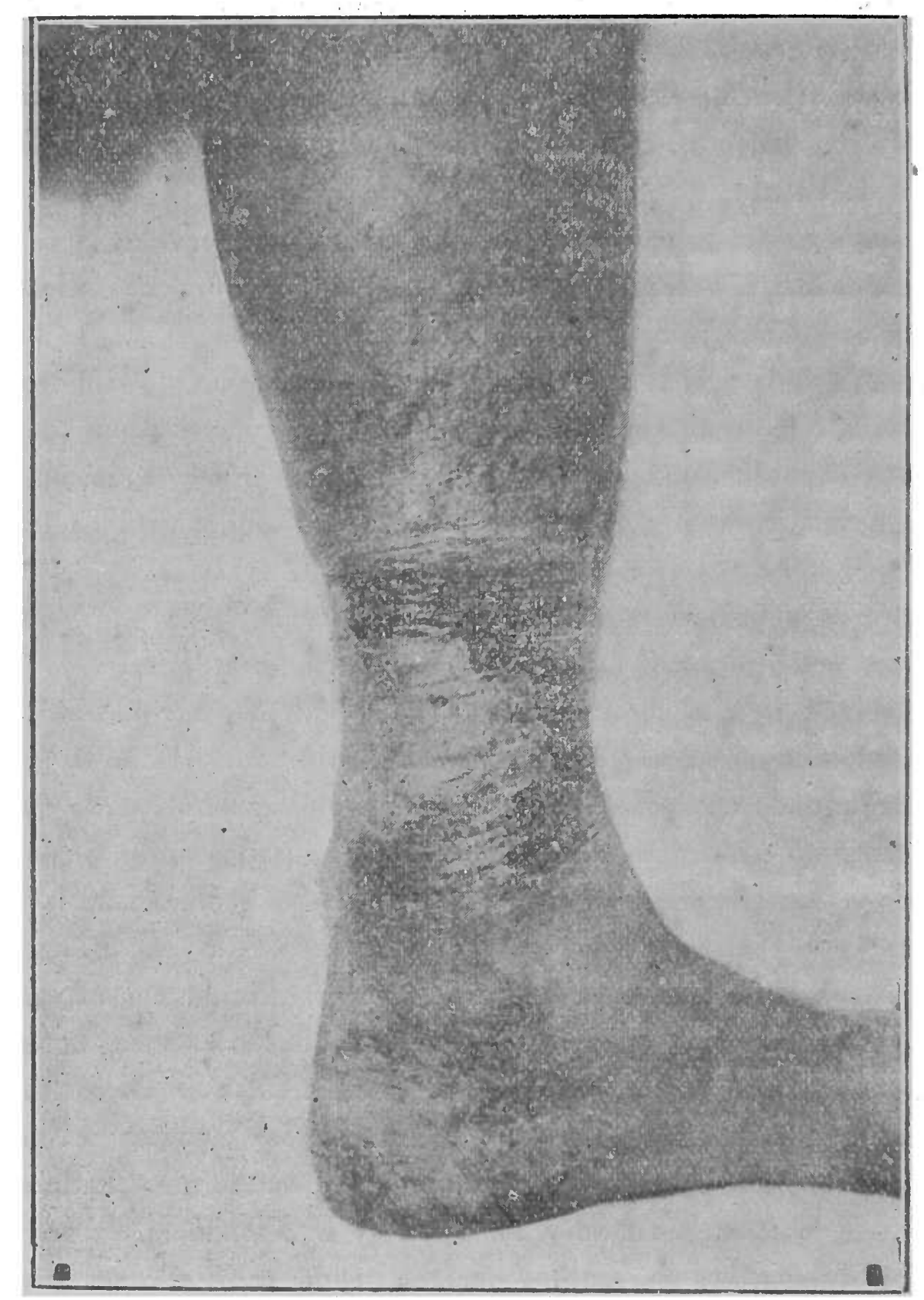

Observação 1-Nota-se a extensa cicatriz após a cura pelo sb. 211.

Para a cura das lesões das mucosas tem sido empregado pelo methodo do Dr. Mario Ottoni o acido lactico a $80 \%$, o qual, apezar de resultados animadores, apresenta a desvantagem de necessitar de especialistas para o seu emprego. 
O preparado que temos usä́o com magnificos resultados trata-se como acima dissemos, do Sb 211.

Apresenta uma reacção francamente acida. Euma combinação ccmplexa, facilmente soluvel, em agua, a qual sendo uma substancia em pó, amorpho, produz em soluçãa a coloração violacea dos saes anti-, moniaes.

O conteúdo de antimonio é de $26,4 \%$. A sua toxidez corresponde ao conteúdo em antimonio e é mais ou menos a mesma que no tartaro emetico.

Nos casos que logo passaremos a desrever, o soluto usado foi feito na porcentàgem de $1 \%$ em agua distillada, e a dose therápeutica usada variando de 0,10 cc. a 0,20 cc.

\section{$N \cdot{ }^{\circ} 1$}

\section{OBSERVAÇÕES}

A. E., solteiro, lavrador, procedente de Araçatuba, côr branca, com 35 annos de idade, portador de leshmaniose verrucosa no terço inferior, parte posterior, da perna. (vide clichés, nos quaes se nota a enorme cicatriz produzida). Estava doente ha dois annos, mais ou menos, não tendo conseguido melhoras com o tartaro emetico, devido ás fortissimas dôres rheumatoides que tal tratamento produzia.

Iniciou o tratamento em 1 de Dezembro de 1925, e teve alta, completamente curado em 5 de Maio de 1926.

Quantidade de medicamento usado: 5 grs., 35.

TRATAMENTO:

Dezembro de 1925

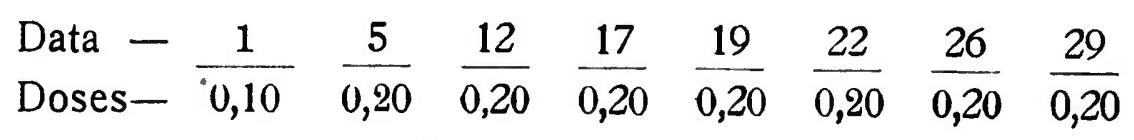

Janeiro de 1926

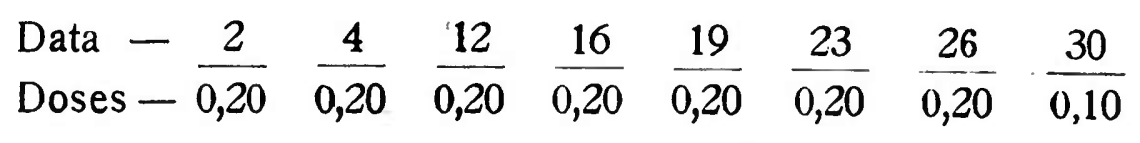

Fevereiro de 1926

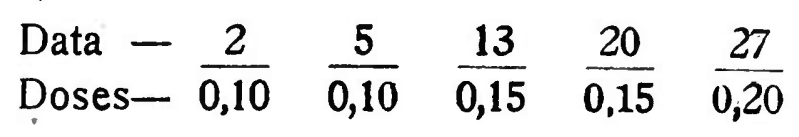


Março

$$
\begin{array}{llllll}
\text { Data }-\frac{6}{0,20} & \frac{13}{0,15} & \frac{20}{0,10} & \frac{23}{0,10} & \frac{27}{0,10} & \frac{30}{0,10}
\end{array}
$$

Abril

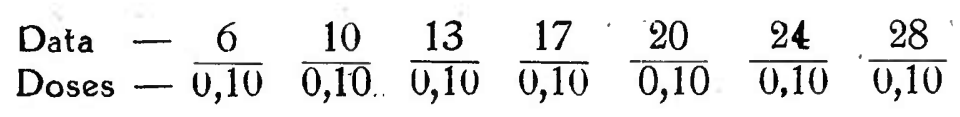

Màio

\section{N. ${ }^{\circ} 2$}

$$
\begin{array}{ll}
\text { Data }-\frac{1}{0,10} & \frac{4}{0,10}
\end{array}
$$

J. A., 45 annos, solteiro, laviador, procedente de Albuquerque Lins, preto. Diagnostico: leishamniose naso-pharingeana.

Anteriormente ao tratamento pelo $\mathrm{Sb} 211$, fez durante muitos mezes 0 . tratamento pelo tartaro sem resultado algum. $O$ nosso tratamento pelo Sb 2 II , foi iniciado em $1 .^{\circ}$ de outubro de 1925 e teve alta curado em 26 de abril de 1926, como attesta o lattestado do dr. Mario Ottoni. Este doente está completamente são tanto do nariz quanto do nasopharinge, bocca, e sobretudo da laringe em que o processo cicatricial é simplesmente admiravel: Está absolutamente curado de sua leishmaniose.

Este doente fazia como os demais injecções 2 vezes por semana pela via endovenosa.

Quantidade de medicamento usado: 6 grs., 17.

Tratamento:

Outubro de 1925

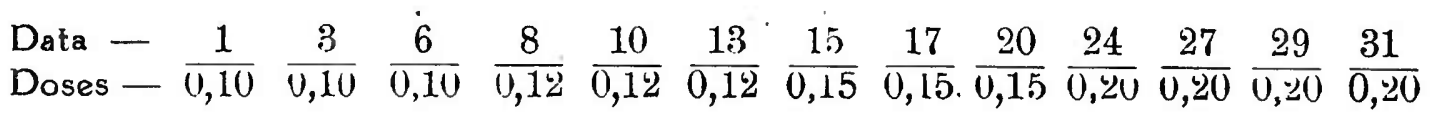

Novembro de 1925

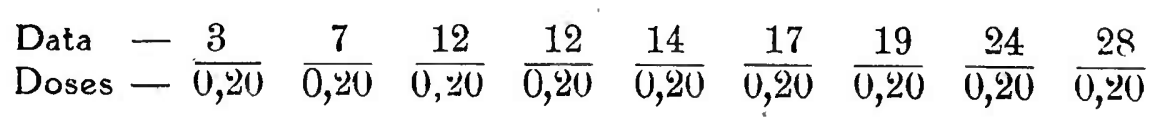

Dezembro

$$
\begin{aligned}
& \text { Data } \\
& \text { Doses }-\frac{1}{0,10}
\end{aligned} \frac{5}{0,10} \frac{12}{0,10} \frac{17}{0,10} \frac{19}{0,10} \frac{22}{0,10} \frac{26}{0,10} \frac{29}{0,10}
$$

Janeiro de 1926

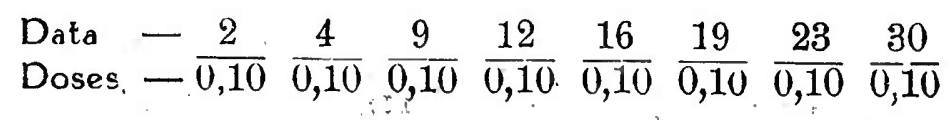




\section{Fevereiro}

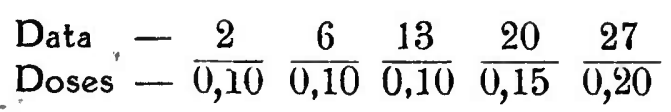

Março

Abril

$$
\begin{aligned}
& \text { Data }-\frac{6}{0,20} \frac{13}{0,20} \cdot \frac{20}{0,20} \frac{23}{0,20} \frac{27}{0,20} \frac{30}{0,20}
\end{aligned}
$$

$$
\begin{aligned}
& \text { Datã }-\frac{6}{0,20} \frac{10}{0,20} \frac{13}{0,20} \frac{17}{0,20} \frac{20}{0.20} \frac{24}{0,10}
\end{aligned}
$$

N. ${ }^{3} 3$

D. F. G., 48 annos, solteiro, lavrador, branco, brasileiro, procedente de JugueryıMirim. Diagnostico: leishmaniose nasal. .Fez algumas injecções de tattaro. Iniciou seu tratamento pelo 211 em 1.c de outubro e teve alta curado em 15 de fevereiro de 1926. Mucosa cicatrisada.

Quantidade de medicamento usado: 4 grs., 95.

Outubro de 1925

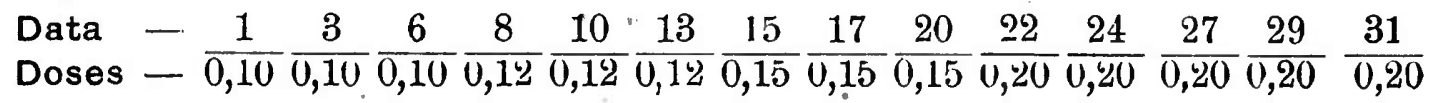

:Novembro

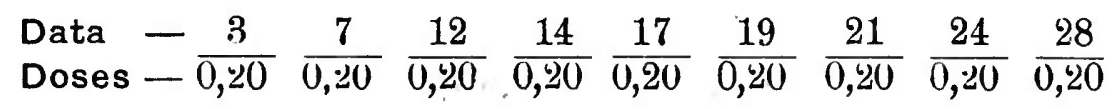

Dezembro

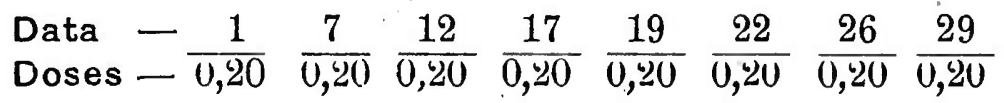

Jameiro de 1926

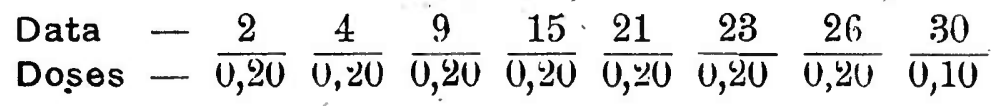

Fevereiro

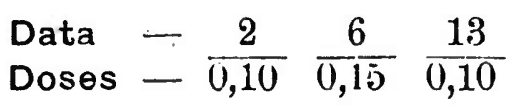

$\mathrm{N} .^{\circ} 4$

J. M., 30 annos, solteiro, preto, brasileiro, lavrador, procedente da Noroeste.

Diagnostico: leishmaniose nasal verrucosa do nariz. 
Foi curetado cinco vezes. Iniciou o tratamento pe 211 em 12 de Novembro de 1925 e teve alta curado em 11 de Março de 1926:

Quantidade de medicamento usado: 3 grs., 95.

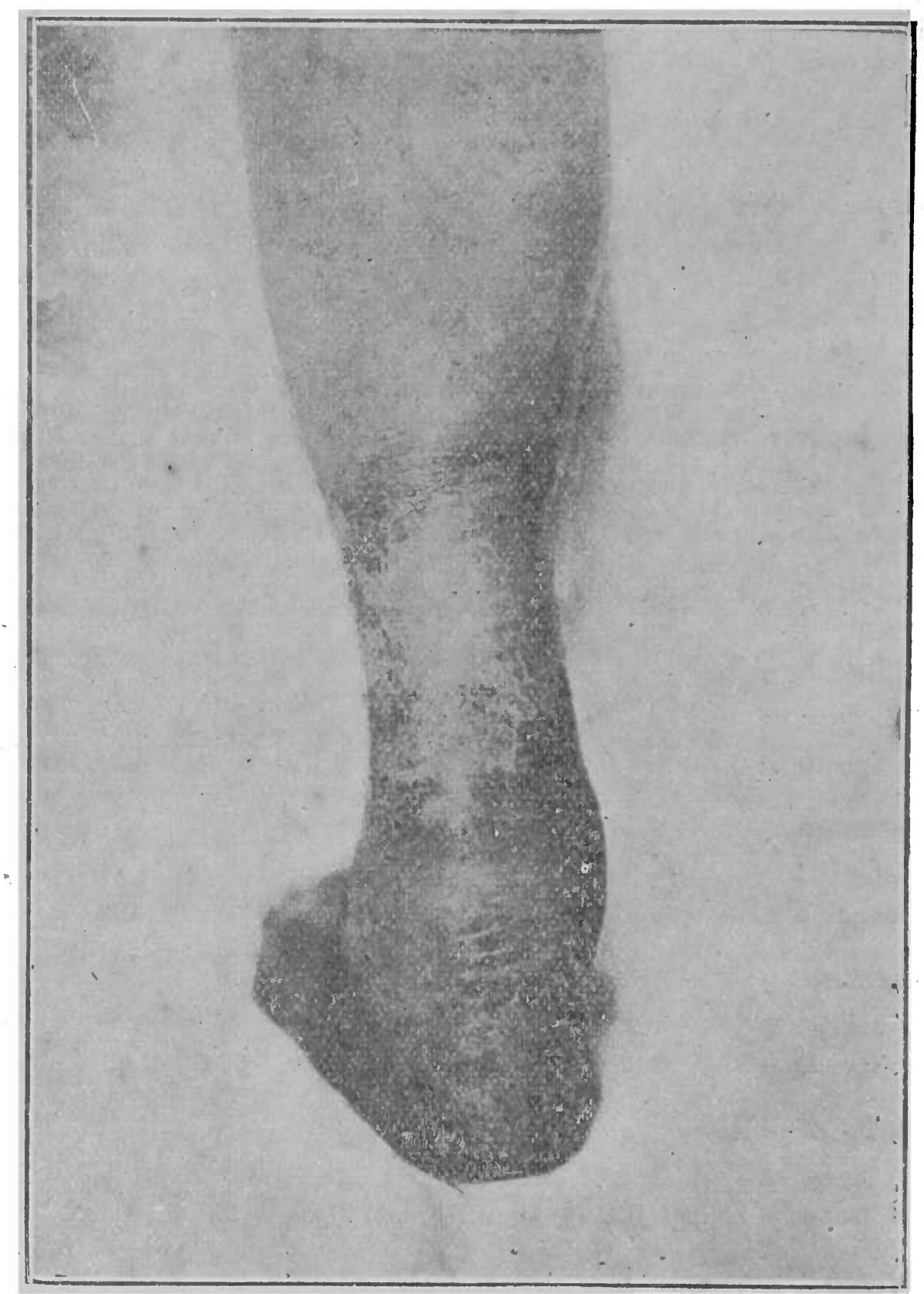

- mesmo doente em outra posição para que se tenha idéa da extensão da cicatriz TRATAMENTO:

Novembro

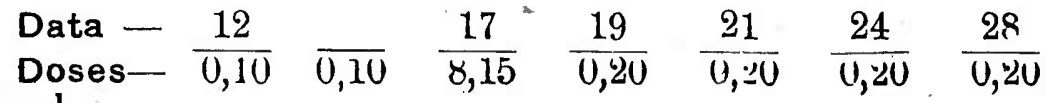

$$
\begin{aligned}
& \text { Dezembro } \\
& \begin{array}{l}
\text { Data }- \\
\text { Doses }-\frac{1}{0,20}
\end{array} \frac{5}{0,20} \quad \frac{19}{0,20} \cdot \frac{17}{0,20} \quad \frac{19}{0,20} \quad \frac{23}{0,20} \cdot \frac{26}{0,20} \quad \frac{29}{0,20}
\end{aligned}
$$


Janeiro de 1926

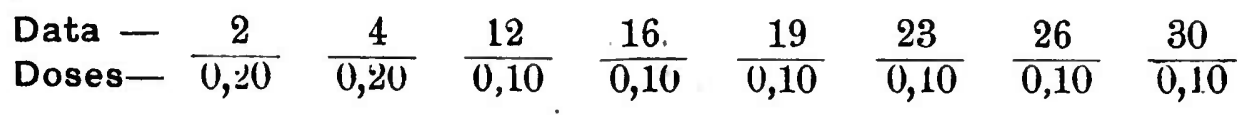

Fevereiro

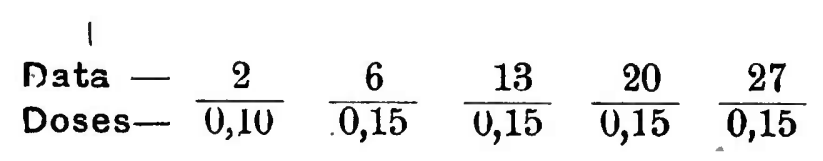

Março

$$
\begin{aligned}
& \text { Data }-\frac{6}{0,15} \\
& \text { Doses- }
\end{aligned}
$$

N.o 5 .

G. A., 20 annos, solteiro, branco, brazileiro, lavrador, procedente Juliapolis, Diagnostico: leishmaniose nasal.

Iniciou o tratamento em 31 de outubro de 1925 e teve alta por curado em 10 de Março de 1926.

Quantidade de medicamento usado: 5 prs., 65.

Novembro de 1925.

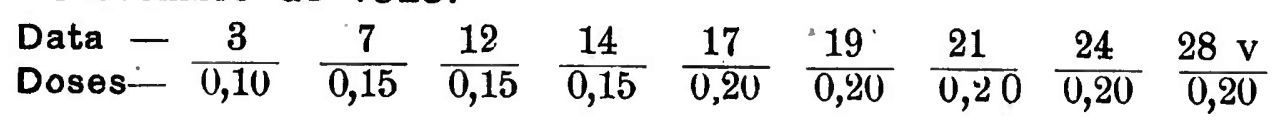

\section{Dezembro}

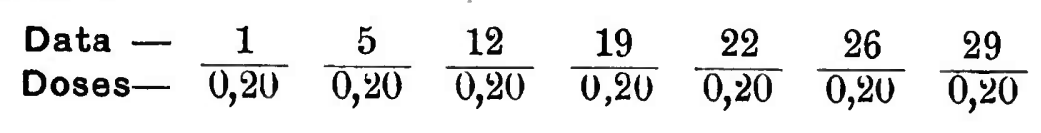

Janeiro de 1926

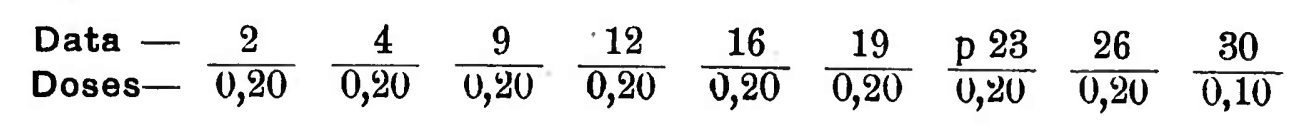

Fevereiro

$$
\begin{array}{llll}
\text { Data }-\frac{2}{0,10} & \frac{6}{0,15} & \frac{20}{0,15} & \frac{27}{0,20}
\end{array}
$$

Março

$$
\begin{aligned}
& \text { Data }-\frac{6}{0,20} \\
& \text { Doses- }
\end{aligned}
$$


I. V., com 25 annos, casada, brasileira, secundipara.

Primeirc parto a termo, normal. Puerperio bom. Segundo parto, em 21 de Abril de 1926, prematuro (8 mezeis). O. E.'A. Dequitadura artificial, hemorrhagia, tentativa de descolamento pela manobra de Credé e tracções sobre o funiculo. Continuação de hemorrhagia, anemia grave (mucosas descoradas, pulso filiforme, nauseas, vomitos, perdı dos sentidoŕ). Desinfeç̧ão previa com alcool e iodo. Luvas esterelisadas. Descolamento manual, notando-se então encarceramento parcial da placenta no angulo uterino direito. Pituitrina. Ergotina. Formação do globo de isegurança. In'jecção de sôro glycorsado adrenalisado: 1 litro.

No dia seguinte, 22 de Abrli, apyrexia, pulso alot. Locchios normaes. Quatro dias após, 26, calefrios intensos, temperąura $40^{\circ}$, dores no flanco direito. Annexo direito espessido. Bolsa de gelo. No quinto dia, calefrics, temperatura elevada. Estado geral máu. Inicio da vaccinotherapia endovenosa.

Exame de urina - pesquisa de pús e coli-bacillo: nagativo.

Hemocultura - negativa.

Exame dos lochios - estreptococcus e erítaphylococcus.

Apparelho digeistivo e pulmonar normaes, o mesmo acont?cendo com o apparelbo cardio vascular.

DIAGINOSTICO CLINIICO: — Infecção puerperal.

\section{TRATAMENTO}

No dia seguinte, 22 de Abril, apyrexia, puliso alto. Lopchios Antipiógeno Polivalente Bruschettini, ás 8 horas da noite, na dóse de 5 cc. No dia fieguinte, pəla manhã,. fizemos applicação de 6 cc. e, á noite, nova injeção de $10 \mathrm{cc}$. No dia 29 de A.bril, pela mıanhã, applicamo, nova injecção de Vaccina Antipiógena Polivalente Bruschettini na dosagem de 12 cc. Como a nossa doente não apresentasse melhoras, resolvemos fazer o aibcesso de fixação. A'. noite, a doente apresentava isensiveis melhoras; o abcesso, apresentava-se rubro e muito dolorido. Fizemos nessa mesma noite nova applicação de $12 \mathrm{cc}$. de "Vaccino Antipiogeno Polivalente Bruschettin"i No dia 30 , pəla manhã, a doente apressentava 37,5 de temperatura e pulso 100 . Nova applicação de $12 \mathrm{cc}$. de "Vaccino Antipiogeno Polivalente Bruschettini". No dia 30 , á noite, a nossa doente aprosentava temperatura e pulso elevados, injectamos 12 cc. de "Vaccino Antipiogeno Polivalente Bruschettini". No dia 1 de Maio, pəla manhã, fizemos nova applicação de $12 \mathrm{cc}$. de "Vaccino Antipiogeno Polivalentie Bruschettini", abrimos o abcesso e drenamos com gaze com o mesmo liquido que serviu para provocar o abcesso. No dia 1 de Maio, pela noite, a deente apresentava melhoras bastante apparientes No dia seguinte encontramors a nosisa doænte sem febre e bem disposta. Começamos a diminuir a dóse da Vaccina e injectamos $10 \mathrm{cc}$. pela manhã é $8 \mathrm{cc}$. pela noite. Nos dias 3 ie $4 \mathrm{~d}$ e Maio applicamos $6 \mathrm{cc}$. pela manhã e $6 \mathrm{cc}$. pela noite; a doente não apresentavia maỉis febre. 0 pulso era normal.

Estado geral bom. Nos dias 6 e 7 de Maio fizemos 6 ce. de "VVaccino Antipiogeno Polivalente Bruschettini", sómənte pela manhã. No dia 8 de Maio demos alta, curada.

As injecções de "Vaccino Antipiogeno Polivalente Bruschettini" for am precedidas de injieçõos de oleo camphorado, eriparteina, cafeina, sendo todas por via endovenosa. No dia. 15 de Maio a nosisa doente vae ao nosso escriptoric, comprimentar-nos, completamente boa, istado geral optimo, revelando pelo toque utero normalmente involuido. 


\section{Conclusões}

Em conłclusão, o tratamento pelo Sb 211 Bayer, apresenta vantajens porque:

não produz como o tartaro hypodermicamente, irritaçães, nem intramuscularmente inflamações, nem en:dovenosamente, as conhecidas devastações dos vasos:

não exige como o tratamento pelo acidó lactico de ser manejado sómente por especialistas;

de produzir em um tempo incomparavelmente menor, a cicatrização das lesões leishmanioticas;

exerce sua acção cicatrizante quer sobre as lesões tegumentares como sobre as das mucosas;

não produzir șeu emprego phenomenos de intolerancia antimonial, quer sejam os imediatos, como tosse, nauseas, dores de cabeça, ou tardios como dores rheumatoides, musculares, musculares, ou articulares e muito menos estado syncopal e pulso filiforme.

Com isto não julgamos que esteja resolvido o problema do tratamento da leishmaniose e assim jugulado o mal que infelizmente se vae alastrando de uma maneira impressionante e affastando da lavoura braços de que tanto necessitamos, pois em regra geral são estes os nidividuos mais flagellados, mas temos a convicção de que mais um passo damos aperfeiçoando o tratamento, para a solução final que já se nos accena promissora.

JOSE DE ALCANTARA MADEIRA Interno do Serviço de Dermatologia e Syphiligraphia da Faculdade de Medicina de S. Paulo.

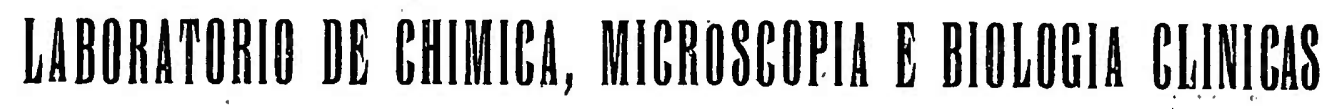
ANALYSES EM GERAL - VACCINOTHERAPIA

Dr. Oscar M. de Barros

Dr. Mendonça Cortez

RUA DIREITA, 35 - 1.0

Telephone: Central, 5033

Caixa Postạl, 1600

SÃo PAULO 